\title{
Proteomic characterization of the cytotoxic mechanism of gold (III) porphyrin 1a, a potential anticancer drug
}

\author{
Ying Wang ${ }^{1,2}$, Qing-Yu He ${ }^{1,2}$, Chi-Ming Che ${ }^{1,2}$ and Jen-Fu Chiu ${ }^{2,3}$ \\ ${ }^{1}$ Department of Chemistry, University of Hong Kong, Pokfulam, Hong Kong SAR, China \\ ${ }^{2}$ Open Laboratory of Chemical Biology, Institute of Molecular Technology for Drug Discovery \\ and Synthesis, University of Hong Kong, Pokfulam, Hong Kong SAR, China \\ ${ }^{3}$ Department of Anatomy, University of Hong Kong, Pokfulam, Hong Kong SAR, China
}

There has been increasing interest in the potential applications of gold (III) complexes as anticancer drugs with higher cytotoxicity and fewer side effects than existing metal anticancer drugs. Our previous findings demonstrated that gold (III) porphyrin 1a preferentially induced apoptosis in a cancer cell line (SUNE1). In this study, we identified differentially expressed proteins related to the drug's cytotoxic action by comparing the protein alterations induced by gold (III) porphyrin 1a and cisplatin treatments. Several clusters of altered proteins were identified, including cellular structure and stress-related chaperone proteins, proteins involved in reactive oxygen species and enzyme proteins, translation factors, proteins that mediate cell proliferation or differentiation, and proteins participating in the internal degradation systems. Our results indicated that multiple factors leading to apoptosis were involved in drug cytotoxicity in SUNE1 cells. The balance between pro-apoptotic and anti-apoptotic signals determined the final fate of cancer cells.

\section{Keywords:}

Anticancer drug/ Apoptosis / Gold compound / Protein profiling / Proteomics
Received: December 4, 2004 Revised: March 23, 2005 Accepted: May 31, 2005

\section{Introduction}

In the past few decades considerable effort has been devoted to the search for new chemotherapeutic agents with preferential anticancer activity. However, although there have been important developments in the field of anticancer che-

Correspondence: Professor Jen-Fu Chiu, Department of Anatomy, Department of Chemistry, University of Hong Kong, Pokfulam, Hong Kong SAR, China

E-mail: jfchiu@hkucc.hku.hk

Fax: +852-2817-1006

\begin{abstract}
Abbreviations: CacyBP, calcyclin binding protein; Cisplatin, cisdiamminedichloro-platinum (II); CLIC 1, chloride intracellular channel protein 1; DAPI, 4, 6-diamidino-2-phenylindole; GDI, rho GDP dissociation inhibitor alpha; IC50, half-maximal inhibitory concentration; $\mathbf{m t S S B}$, mitochondrial single-stranded DNA binding protein; NBB, naphthol blue black; Op18, oncoprotein 18; PDI, protein disulfide isomerase 1; ROS, reactive oxygen species; VDAC1, voltage-dependent anion channel 1
\end{abstract}

motherapy, no breakthrough has been achieved in the search for better anticancer agents since the introduction of cis-diamminedichloro-platinum (II) (cisplatin) into clinical use $[1,2]$. In recent years research has increasingly focused on the potential of gold (III) complexes as anticancer drug candidates, because gold (III) possesses the same isoelectronic configuration $\left(\mathrm{d}^{8}\right)$ and structural (square planar) characteristics as platinum (II) [3-5]. Our laboratories have been attempting to develop gold (III) meso-tetraarylporphyrins as potential chemotherapeutic leads [6]. We have previously reported that one of these complexes, gold (III) meso-tetraarylporphyrin 1a [gold (III) porphyrin 1a] (Fig. 1A), is a promising new chemotherapeutic lead ([6], and to be published). In this study we used a proteomic approach to further investigate the action mechanism of gold (III) porphyrin 1a in suppressing cancer cell growth. Cisplatin was chosen as a control drug because it is one of the most widely used metalcontaining anticancer drugs, and the action mechanism of cisplatin-induced apoptosis has been well documented [7, 8]. In addition, the drug resistance and side effects of cisplatin 
A

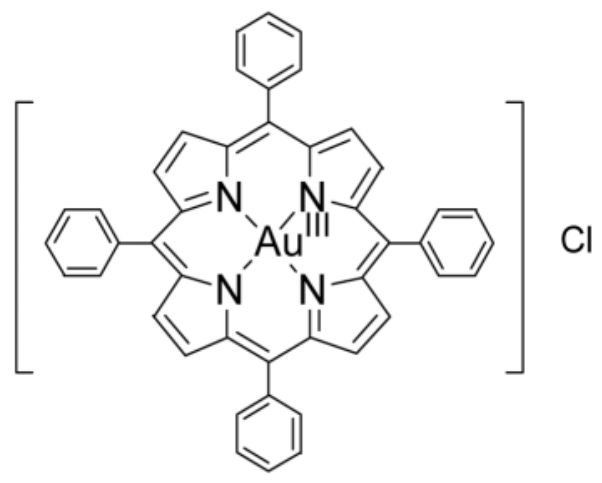

Gold (III) porphyrin 1a

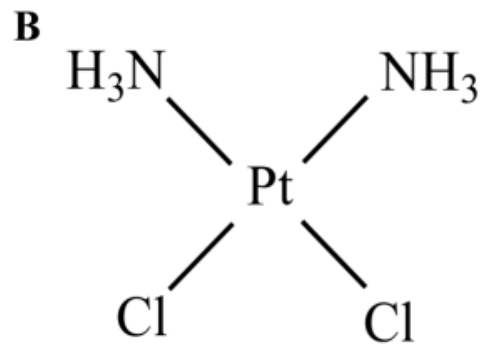

Cisplatin

Figure 1. Chemical structures of gold (III) porphyrin 1a (A) and cisplatin (B).

still pose major problems for its clinical use $[9,10]$. There is an urgent need for the development of new anticancer drug candidates to replace cisplatin. This is particularly important for patients who respond poorly to cisplatin treatment.

Previous studies have indicated that apoptosis induced by interaction with DNA is an important mechanism by which chemotherapeutic agents kill susceptible cells [11]. For example, cisplatin causes apoptosis through cross-linking with DNA [7, 8], and bleomycin induces apoptosis by DNA fragmentation $[12,13]$. Apoptosis, or programmed cell death, is a type of cell death involving characteristic morphological and biochemical changes. This process is achieved via the aggregation and multimerization of the upstream molecules of the death effector that activate sequential pathways. These pathways play pivotal roles in the regulation of cellular physiological and pathological conditions in response to chemotherapeutic cytotoxicity $[14,15]$. Recent studies have made important progress in identifying key molecular components of this intracellular suicide program $[16,17]$. As proteins are the key mediators of cellular activities, the alteration of cellular protein profiles after treatment with chemotherapeutic agents could provide valuable information to improve our understanding of the cytotoxic mechanism of anticancer agents [18].

The relatively recent discipline of proteomics involves the study and characterization of the protein products of the genome, known as the proteome [19]. The scope of proteomics also includes the analysis of protein cellular activities and functions, including the characterization of the flow of information within the cell. Some of this information is provided by protein networks organized in discrete signal transduction pathways, which control cell apoptosis [20]. Proteomic profiling therefore offers a good chance of identifying the proteins that mediate the apoptosis pathways involved when cells are treated with chemotherapeutic agents [21]. By comparing the protein profile of SUNE1 cancer cells treated by gold (III) porphyrin 1a (Fig. 1A) and cisplatin (Fig. 1B), differentially expressed proteins were identified by PMF, and further confirmed by Western blot analysis. Detailed functional analyses of these altered proteins have elucidated the action mechanisms of gold (III) porphyrin 1a and confirmed its potential as an anticancer drug.

\section{Materials and methods}

\subsection{Materials and reagents}

Gold (III) porphyrin 1a was synthesized and purified by a method described previously [6]. Cisplatin was purchased from Sigma-Aldrich Chemical Co. All other chemicals, except where specifically noted, were purchased from SigmaAldrich Chemical Co. and Amersham Biosciences (Uppsala, Sweden). Gold (III) porphyrin 1a and cisplatin were dissolved in DMSO at concentrations of $1 \mathrm{~mm}$ and $30 \mathrm{~mm}$, respectively, and kept as stock solutions.

\subsection{Cell lines and cell culture}

The SUNE1 cell line, a human nasopharyngeal carcinoma (NPC) cell line derived from poorly differentiated NPC in Chinese patients [22], and the NP 69 cell line, the normal nasopharyngeal cell line, were used in this experiment. Both cell lines were generously provided by Prof. S. W. Tsao (Department of Anatomy, The University of Hong Kong). The SUNE1 cell line was cultured in RPMI 1640 medium with $2.0 \mathrm{~g} / \mathrm{L}$ sodium bicarbonate plus $10 \%$ fetal bovine serum (JRH Bioscience, Lenexa, KS), $2 \mathrm{mmol} / \mathrm{L}$ l-glutamine, $100 \mathrm{U} / \mathrm{mL}$ penicillin, and $100 \mu \mathrm{g} / \mathrm{mL}$ streptomycin (GibcoBRL, Grand Isle, NY, USA). The NP 69 cell line was cultured in Keratinocyte-SFM (GIBCO) medium plus supplements for Keratinocyte-SFM (GIBCO). Both cell lines were maintained in a humidified incubator with an atmosphere of $95 \%$ air and $5 \% \mathrm{CO}_{2}$ at $37^{\circ} \mathrm{C}$. 


\subsection{Cytotoxicity assays}

The cytotoxicity of gold (III) porphyrin 1a was determined by Naphthol Blue Black (NBB) staining assay, according to a method previously reported [23]. The NP 69 cells were changed to RPMI 1640 medium the day before the assay to minimize the effects of different contents in the media on the cytotoxicity of the analyzed drugs. Both the SUNE1 cells and the NP 69 cells were plated in 96-well plates at $2 \times 10^{4} /$ well in complete media and incubated overnight. The media were then changed and the cells were grown in media containing various amounts of gold (III) porphyrin 1a and cisplatin respectively for $24 \mathrm{~h}$. At the end of the experiment, the media were removed and the cells were stained by NBB staining assay [23] and measured at $595 \mathrm{~nm}$ using a Model E1 310 Autoplate reader (Bio-Tek Instruments, Winooski, VT).

\subsection{Confocal imaging of morphological changes}

To detect morphological changes in the apoptotic process, nuclear staining was performed by staining with $1 \mu \mathrm{g} / \mathrm{mL} 4$, 6-diamidino-2-phenylindole (DAPI) [24], and cells were analyzed with a fluorescence microscope (Olympus IX71 CTS Chinetek Scientific Microscope).

\subsection{Flow cytometric analysis of cell cycle}

Gold (III) porphyrin 1a-induced apoptosis was determined with flow cytometry according to the method previously described [23]. Cells were cultivated for $24 \mathrm{~h}$ before either left untreated or treated with $1 \mu \mathrm{m}$ gold (III) porphyrin 1a or $30 \mu \mathrm{M}$ cisplatin for $24 \mathrm{~h}$, respectively. This dose was about the $\mathrm{IC}_{50}$ value (half-maximal inhibitory concentration) of each agent. At the end of each experiment, cells were harvested, resuspended in PBS solution, stained by propidium iodide (PI), and analyzed with a FACStar Plus flow cytometer. For each sample $1 \times 106$ cells were analyzed, providing a solid statistical basis for the determination of the percentage of apoptotic cells in each treatment using the Lysis II software program.

\section{$2.62-D E$}

2-DE was carried out with Amersham Biosystems Ettan ${ }^{\text {TM }}$ IPGphor $^{\text {TM }}$ IEF System and Ettan ${ }^{\text {TM }}$ Daltsix Tank (18-cm) units, in accordance with a protocol previously described [25]. Protein samples $(150 \mu \mathrm{g})$ were extracted from cells treated with $1 \mu \mathrm{m}$ gold (III) porphyrin 1a or $30 \mu \mathrm{m}$ cisplatin, and untreated cells were used as control. Triplicate electrophoreses were performed to ensure reproducibility. All gels were visualized by silver staining [25].

\subsection{Image analysis and MS PMF}

Image acquisition and analysis were performed with an Image Scanner (Amersham) and Image Master 2D Elite software (Amersham) [25]. Comparisons were made between gel images of protein profiles obtained from cells treated with gold (III) porphyrin 1a or cisplatin and images from untreated cells. Altered protein spots that changed consistently and significantly (greater than twofold difference) were selected for analysis with MALDI-TOF MS. These protein spots were cut out in small pieces and subjected to in-gel tryptic digestion overnight [25]. Peptide mass spectra were recorded and previously described parameters for spectral acquisition were used [25]. Protein identification with $25 \mathrm{ppm}$ or lower mass error and MOWSE scores over 300 were obtained in most analyses during database matching using MS-Fit (http://prospector.ucsf.edu/). Duplicate or triplicate runs were made to ensure the accuracy of analyses.

\subsection{Western blot analysis}

Cells were lysed and protein extraction was used for Western blot analysis [23]. The samples were separated on $12 \%$ SDS gel and electrophoretically transferred to PVDF membrane (Amersham, Buckinghamshire, UK). The membranes were blotted with $5 \%$ skim milk, washed, and probed with primary antibodies against cyclophilin A (CypA), oncoprotein 18 (Op18), protein disulfide isomerase 1 (PDI), rho GDP dissociation inhibitor alpha (GDI), and stratifin (Santa Cruz, CA, USA), respectively. $\beta$-Actin (Santa Cruz) was used as an internal control for protein loading. The membranes were then washed, incubated with corresponding secondary antibodies, and visualized using an enhanced chemiluminescence (ECL) kit (Amersham), in accordance with the manufacturer's recommendations.

\section{Results}

\subsection{Cancer cells are more sensitive to gold (III) porphyrin 1a than normal cells}

According to the results of the NBB staining assay (Fig. 2), the $\mathrm{IC}_{50}$ of gold (III) porphyrin 1a for NP 69 and SUNE1 cells were $5.5 \mu \mathrm{m}$ and $1.5 \mu \mathrm{M}$, respectively, and the $\mathrm{IC}_{50}$ of cisplatin for NP 69 and SUNE1 cells were $47 \mu \mathrm{M}$ and $3900 \mu \mathrm{M}$, respectively. In other words, gold (III) porphyrin 1a was around 4 times more toxic to tumor (SUNE1) cells than to normal (NP 69) cells, while cisplatin was over 82 times more toxic to SUNE1 cells than to NP 69 cells.

\subsection{Gold (III) porphyrin 1a induces apoptosis in SUNE1 cells}

It has been reported that cancer cells undergo apoptosis under cisplatin treatment [26-29]. In this study, nuclear morphologies were obtained in normal and cancer cells after treatment with gold (III) porphyrin 1a or cisplatin for $24 \mathrm{~h}$, assessed by DAPI staining (Fig. 3A). Apoptotic morphological changes were observed in gold (III) porphyrin 1a-treated cancer cells, but no such changes were observed in normal

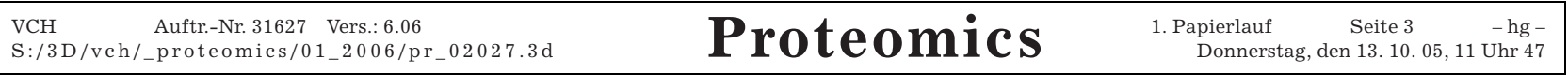




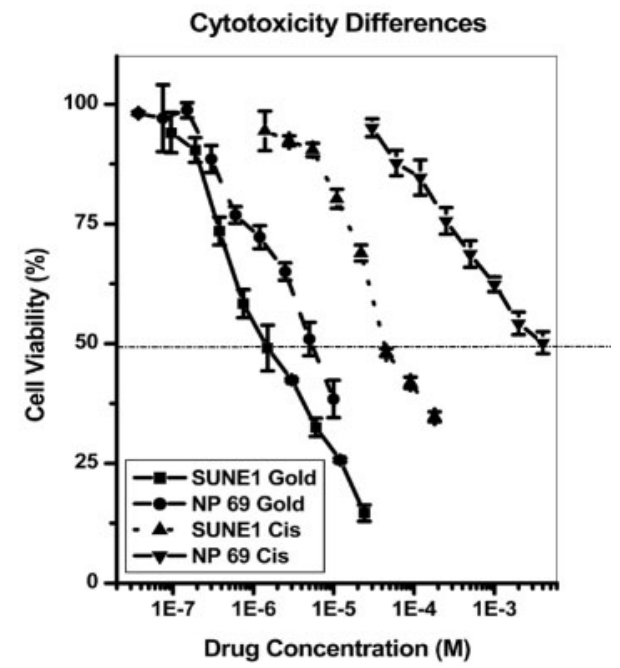

Figure 2. Cytotoxicity differences of gold (III) porphyrin 1a on SUNE1 and NP 69 cells. Gold (III) porphyrin 1a had a greater cytotoxic effect on the SUNE1 cancer cells than on cells of the normal NP 69 cell line under the same condition.
A

P 69

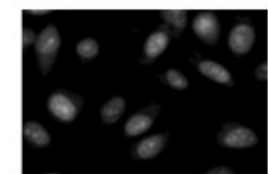

SUNE1

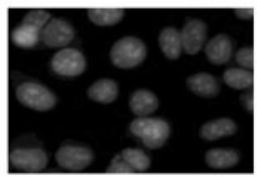

Ctrl
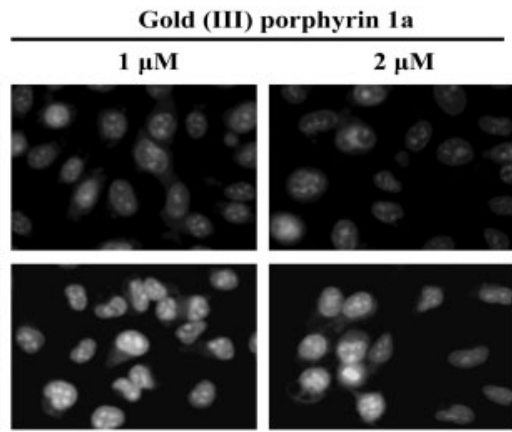
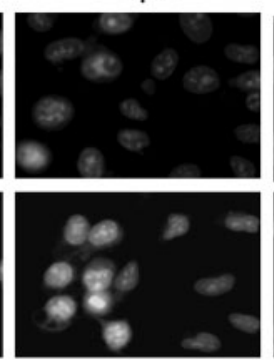

B

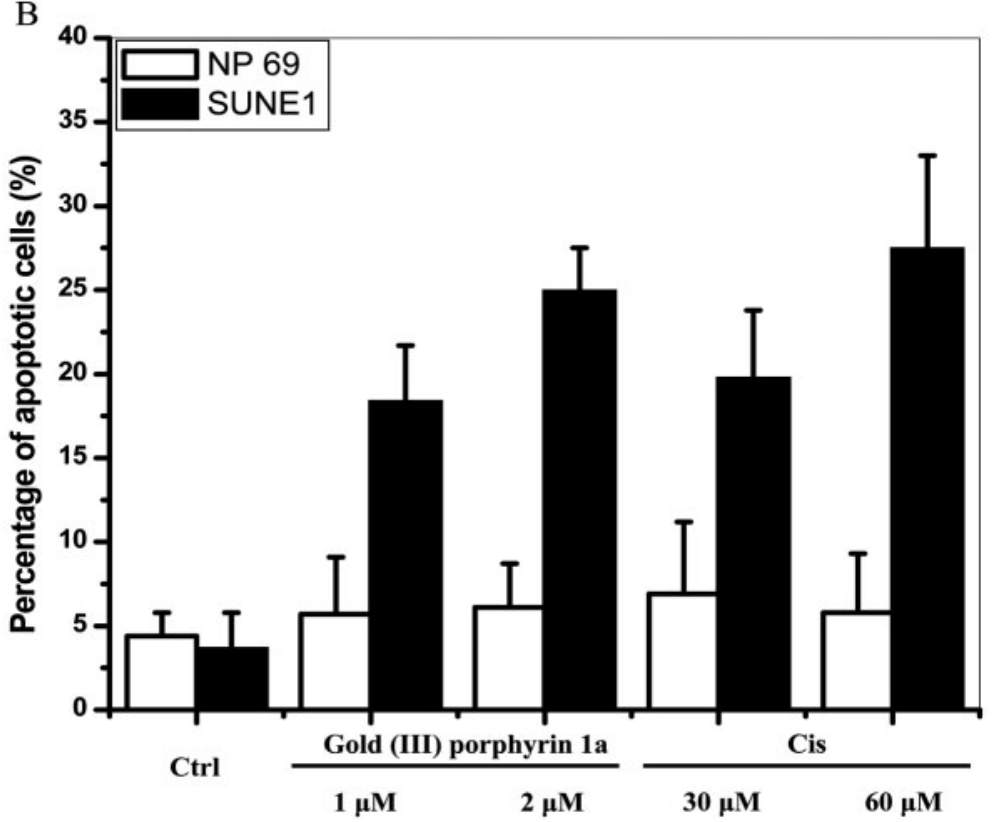

(C) 2005 WILEY-VCH Verlag GmbH \& Co. KGaA, Weinheim cells. The numbers of apoptotic and non-apoptotic cells were quantitated and presented as percentages (Fig. 3B). The effects of gold (III) porphyrin 1a on cell death were further investigated by flow cytometric analysis. The percentage of apoptotic cells in SUNE1 cells after gold (III) porphyrin 1a treatment was dramatically higher than in the control cells (Fig. 4), and this result was in accordance with the DAPI stain results. They also confirmed our previous findings (to be published), that gold (III) porphyrin 1a induces apoptosis in SUNE1 cells.

\subsection{Proteome profiles of control and drug-treated cancer cells}

To further investigate the mechanism of cell apoptosis induced by drugs, protein profiles of control, gold (III) porphyrin 1a- and cisplatin-treated SUNE1 cells were studied by comparative proteomic analysis. Representative 2-D gel images for control, gold (III) porphyrin 1a- and cisplatin-treated SUNE1 cells are shown in Fig. 5A. Figure 5B shows an enlarged image overview for a typical master gel of a SUNE1

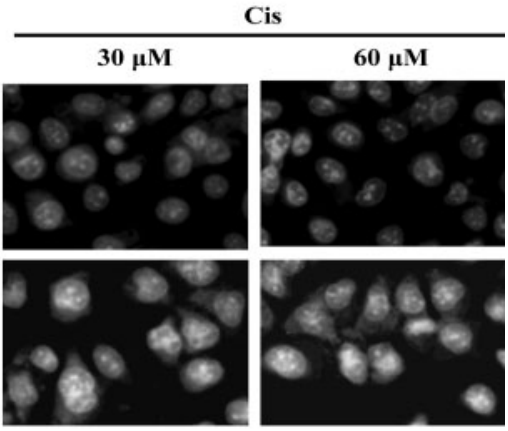

Figure 3. (A) Morphological changes induced by gold (III) porphyrin 1a in SUNE1 and NP 69 cells. The cells were stained by DAPI and visualized by confocal microscope. Typical apoptotic morphological changes were detected in gold (III) porphyrin 1a-treated SUNE1 cells. (B) Percentage of apoptotic and nonapoptotic cells under gold (III) porphyrin 1a and cisplatin treatment in NP 69 and SUNE1 cells.

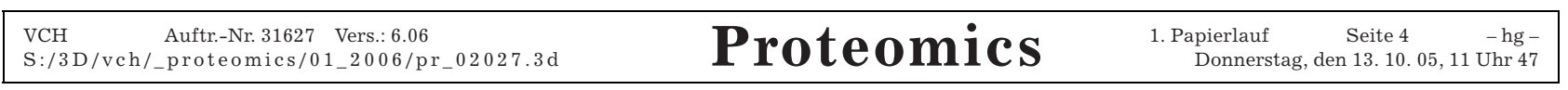



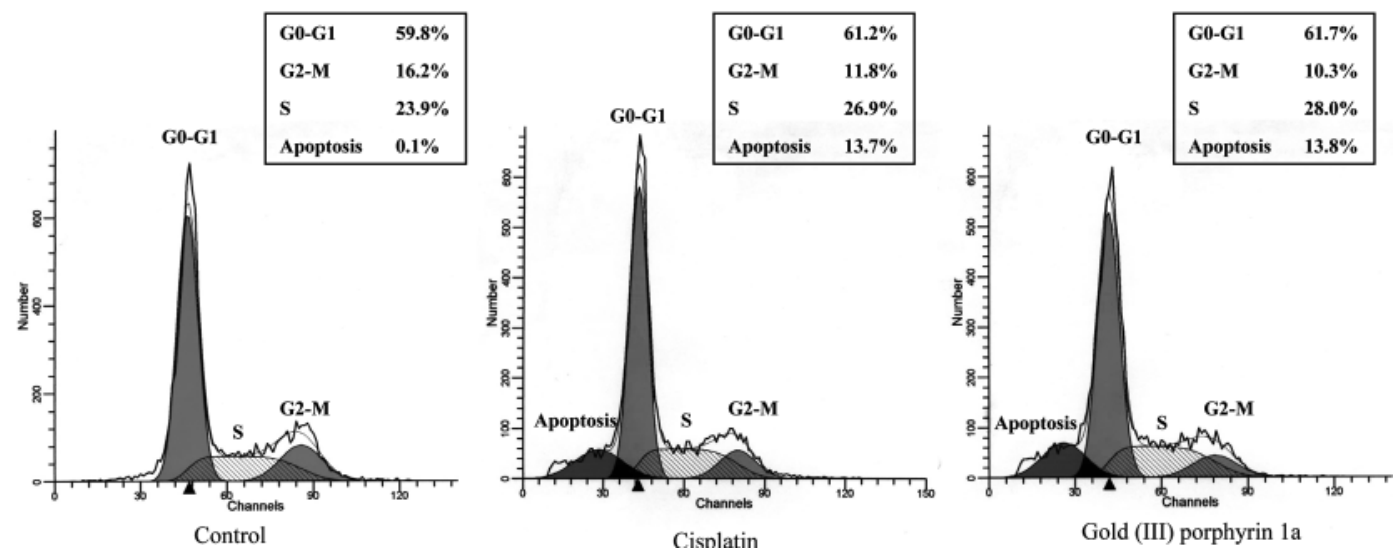

Figure 4. Flow cytometric analysis indicating gold (III) porphyrin 1a-induced apoptosis in SUNE1 cells.

A

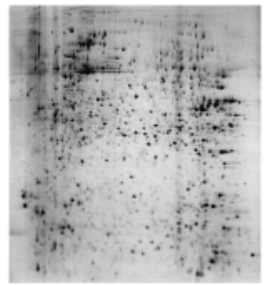

Control

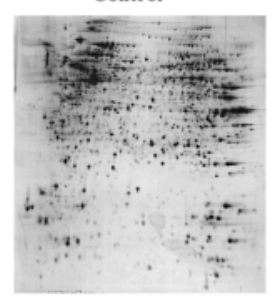

Cisplatin

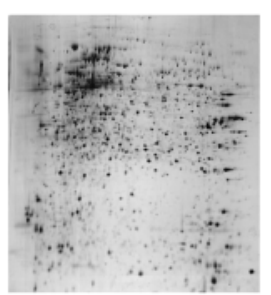

Gold (III) porphyrin 1a
B

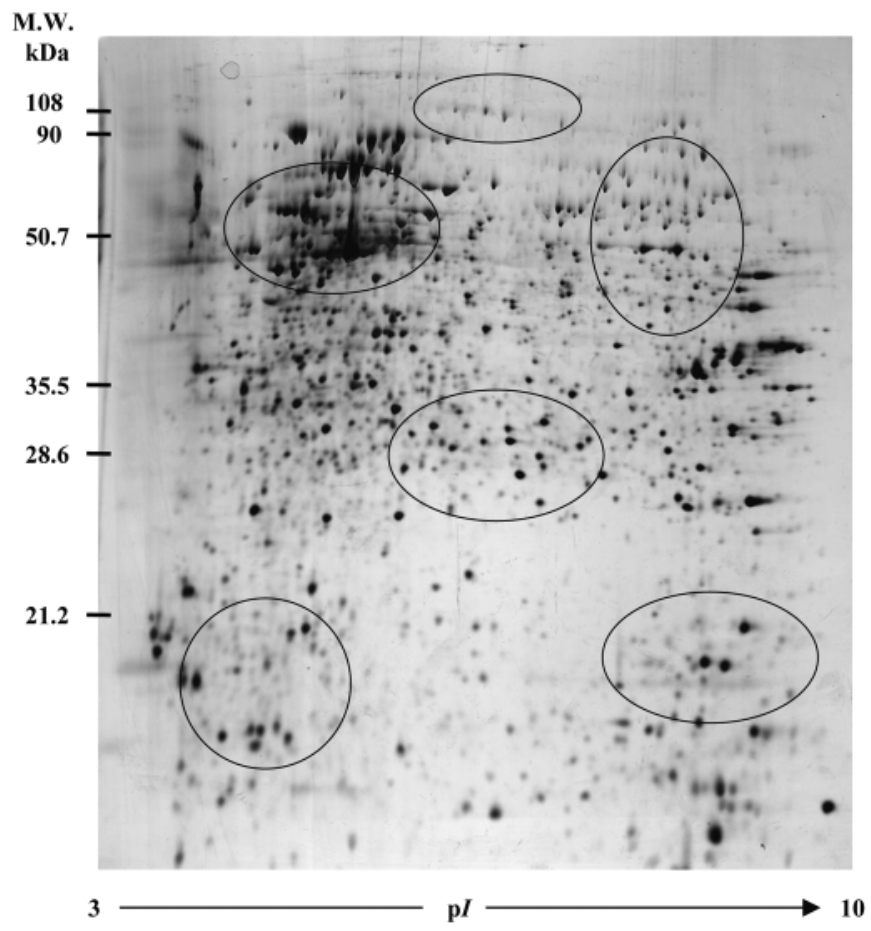

Figure 5. (A) Representative 2-D gel images for control, gold (III) porphyrin 1a- and cisplatin-treated SUNE1 cells (24-h incubation). (B) An image overview of a typical master gel from SUNE1 cells treated with gold (III) porphyrin $1 \mathrm{a}$ for $24 \mathrm{~h}$. Areas where significant and consistent alterations of protein expression were identified are circled.

cell treated with gold (III) porphyrin 1a for $24 \mathrm{~h}$. Over 1000 protein spots were clearly separated on the gels. Circles highlight major areas where significant and consistent alterations of protein expression were identified. These altered proteins were distributed evenly throughout the entire gel, indicating that multiple clusters of proteins are involved in the process of gold (III) porphyrin 1a-induced apoptosis.
Table 1 shows protein ID obtained through PMF, together with proportionate differences of expression and major functions of the identified altered proteins through PMF matching. These proteins can be classified into several categories based on their major biological functions, including cellular structural proteins, stress-related and chaperone proteins, proteins involved in reactive oxygen species (ROS), enzyme proteins and translation factors, proteins that me-

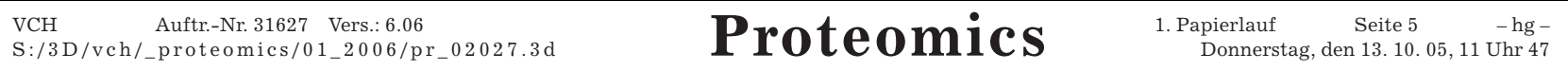


Table 1. Protein alterations in SUNE1 cell under $1 \mu \mathrm{m}$ gold (III) porphyrin $1 \mathrm{a}$ and $30 \mu \mathrm{M}$ cisplatin treatments for $24 \mathrm{~h}$

\begin{tabular}{|c|c|c|c|}
\hline \multirow[t]{2}{*}{ Protein ID } & \multicolumn{2}{|c|}{ Change (fold) } & \multirow[t]{2}{*}{ Major functions } \\
\hline & Gold 1a & Cisplatin & \\
\hline Stomatin-like 2 & +2.3 & +2.3 & Cellular structure protein \\
\hline Lamin C (fragment) & -3.3 & +2.3 & Cellular structure protein, nuclear envelope \\
\hline Heat shock protein gp96 precursor (HSPgp96) & +4.4 & +3.7 & Stress-related, chaperone \\
\hline Peroxiredoxin 1 & +2.9 & +2.5 & Chaperone, and redox activities \\
\hline Peroxiredoxin 6 & +2.1 & +2.5 & Chaperone, and redox activities \\
\hline Thioredoxin & +2.8 & +3.8 & Redox activities \\
\hline $\begin{array}{l}\text { Mitochondrial single-stranded DNA binding } \\
\text { protein (mtSSB) }\end{array}$ & undetected & undetected & Translation factor \\
\hline Splicing factor 17 & +3.8 & +3.7 & Translation factor \\
\hline Protein disulfide isomerase 1 (PDI) & -2.4 & -4.6 & Translation factor, chaperone \\
\hline Triosephosphate isomerase 1 (TPI 1) & -1.1 & +2.4 & Enzyme protein, glycolysis \\
\hline Peptidylprolyl isomerase F, cyclophilin F (CypF) & -3.1 & -3.0 & Enzyme protein, catalysis \\
\hline Oncoprotein 18 (Op18) & -1.4 & +2.9 & Cell proliferation or differentiation \\
\hline Cyclophilin A (CypA) & -2.0 & +1.7 & Cell proliferation or differentiation \\
\hline $\begin{array}{l}\text { Calcyclin binding protein, Siah-interacting } \\
\text { protein (CacyBP) }\end{array}$ & -1.4 & -2.4 & Cell proliferation or differentiation \\
\hline Rho GDP dissociation inhibitor alpha (GDI) & +1.1 & +4.2 & Cell proliferation or differentiation \\
\hline Ras-related nuclear protein & +1.6 & +2.5 & Cell proliferation or differentiation \\
\hline $\begin{array}{l}\text { Porin isoform 1; voltage-dependent anion } \\
\text { channel } 1 \text { (VDAC1) }\end{array}$ & +5.7 & +2.2 & Cell proliferation or differentiation \\
\hline Ionized calcium binding adapter molecule 2 (IBA 2) & +3.2 & +5.1 & Cell proliferation or differentiation \\
\hline Chloride intracellular channel protein 1 (CLIC 1) & +2.3 & +2.5 & Cell proliferation or differentiation \\
\hline Stratifin & +5.9 & +4.4 & Cell proliferation or differentiation, chaperone \\
\hline Proteasome subunit, alpha type 3 & -1.1 & +5.2 & Internal degradation \\
\hline Proteasome subunit, beta type 4 & -1.3 & +4.6 & Internal degradation \\
\hline Proteasome subunit alpha type 6 & +1.6 & +2.6 & Internal degradation \\
\hline
\end{tabular}

diate cell death and survival signaling, and proteins that participate in the internal degradation system. Table 2 summarizes the parameters obtained in database matching for protein identification, including NCBI database access number, sequence coverage, and experimental mass and $\mathrm{p} I$.

\subsection{Protein alterations in the cells treated with gold (III) porphyrin 1a and cisplatin}

Figure 6 illustrates the detailed comparison of proteins involved in cellular structure and oxidative stress. In comparison with the control cells, stomatin-like 2 was significantly up-regulated in both gold (III) porphyrin 1a- and cisplatin-treated cells, while the nuclear envelope protein lamin $\mathrm{C}$ was suppressed in cells treated with gold (III) porphyrin 1a. The up-regulation of stress-related chaperones and redoxrelated proteins, including heat shock protein gp96 precursor (HSP gp96), peroxiredoxin 1 and 6, and thioredoxin was also observed in the cells treated with both agents.

Figure 7 shows the altered expression of translation factors and enzyme proteins. Not all of the proteins in this group were equally regulated. For example, triosephosphate isomerase 1 (TPI1) was down-regulated by gold (III) por- phyrin 1a treatment, but up-regulated by cisplatin treatment. On the other hand, the negative regulation of PDI and cyclophilin F was observed in the cells treated with both agents. Mitochondrial single strand DNA binding protein (mtSSB) was undetectable after treatment by both agents.

Figure 8 displays the signal transduction proteins altered in the cells treated with gold (III) porphyrin 1a. The expressions of Op18 and CypA were suppressed by gold (III) porphyrin 1a but elevated by cisplatin. Calcyclin binding protein (CacyBP), GDI, and Ras-related nuclear protein were regulated in the same manner by both agents. Three ion-related signal transduction proteins, the voltage-dependent anion channel 1 (VDAC1), ionized calcium binding adapter molecule 2 (IBA 2), and chloride intracellular channel protein 1 (CLIC 1), were up-regulated under gold (III) porphyrin 1a treatment. The over-expression of VDAC was much more significant under treatment with gold (III) porphyrin 1a than with cisplatin.

The differential expression of proteins involved in internal protein degradation is shown in Fig. 9. This group of proteins, including proteasome subunit beta type 4, alpha type 3, and alpha type 6, is up-regulated under cisplatin treatment, but shows no significant change under gold (III) porphyrin 1a treatment. 
Table 2. Results from MALDI-TOF mass spectra and database searching for protein identification

\begin{tabular}{|c|c|c|c|}
\hline Protein ID & $\begin{array}{l}\text { Accession } \\
\text { no. (NCBI) }\end{array}$ & $\begin{array}{l}\text { Sequence } \\
\text { coverage }\end{array}$ & $\begin{array}{l}\text { Experimental } \\
\text { mass }(\mathrm{Da}) / \mathrm{p} /\end{array}$ \\
\hline Stomatin-like 2 & 704102 & $26.0 \%$ & 38534 / 6.9 \\
\hline Lamin C (fragment) & 5031875 & $22.0 \%$ & $65135 / 6.4$ \\
\hline Heat shock protein gp96 precursor (HSPgp96) & 15010550 & $17.0 \%$ & $90195 / 4.7$ \\
\hline Peroxiredoxin 1 & 4505591 & $39.0 \%$ & $22111 / 8.3$ \\
\hline Peroxiredoxin 6 & 1718024 & $50.0 \%$ & $25035 / 6.0$ \\
\hline Thioredoxin & 135773 & $45.0 \%$ & $11738 / 4.8$ \\
\hline Mitochondrial single-stranded DNA binding protein (mtSSB) & 4507231 & $30.0 \%$ & $17260 / 9.6$ \\
\hline Splicing factor 17 & 7706657 & $15.0 \%$ & 65522 / 6.6 \\
\hline Protein disulfide isomerase 1 (PDI) & 7437388 & $26.0 \%$ & 56783 / 6.0 \\
\hline Triosephosphate isomerase 1 (TPI 1) & 17389815 & $22.0 \%$ & 26642 / 6.4 \\
\hline Peptidylprolyl isomerase $\mathrm{F}$, cyclophilin F (CypF) & 5031987 & $22.0 \%$ & $22040 / 9.5$ \\
\hline Oncoprotein 18 (Op18) & 5031851 & $42.0 \%$ & $17303 / 5.8$ \\
\hline Cyclophilin A (CypA) & 1334908 & $34.0 \%$ & $23207 / 5.0$ \\
\hline Calcyclin binding protein, Siah-interacting protein (CacyBP) & 5454052 & $23.0 \%$ & 27774 / 4.7 \\
\hline Rho GDP dissociation inhibitor alpha (GDI) & 1351015 & $22.0 \%$ & $24423 / 7.0$ \\
\hline Ras-related nuclear protein & 4507879 & $27.0 \%$ & 30723 / 8.6 \\
\hline Porin isoform 1; voltage-dependent anion channel 1 (VDAC1) & 13899241 & $12.0 \%$ & 17068 / 6.6 \\
\hline Ionized calcium binding adapter molecule 2 (IBA 2) & 14251209 & $26.0 \%$ & $26923 / 5.1$ \\
\hline Chloride intracellular channel protein 1 (CLIC 1) & 7656952 & $21.0 \%$ & $26210 / 8.3$ \\
\hline Stratifin & 5454052 & $23.0 \%$ & 27774 / 4.7 \\
\hline Proteasome subunit, alpha type 3 & 23110939 & $25.0 \%$ & $27648 / 5.2$ \\
\hline Proteasome subunit, beta type 4 & 298507 & $21.0 \%$ & $29192 / 5.7$ \\
\hline Proteasome subunit, alpha type 6 & 8394076 & $28.0 \%$ & $27400 / 6.3$ \\
\hline
\end{tabular}

Western blot analysis was used to confirm the altered expressions of proteins in response to the treatment by these drugs. Since a large number of altered proteins were identified and not all proteins can be confirmed by Western blot analysis, we only selected PDI, GDI, stratifin, Op18, and CypA, because their database matching scores are lower than 200 or they are differentially expressed after treatment by gold (III) porphyrin 1a and cisplatin, respectively. Figure 10 shows the Western blot results of GDI, Op 18, Cyp A, stratifin, and PDI. The altered expression ratio of these proteins matched well with the ratio detected in 2-DE.

\section{Discussion}

\subsection{Tumor targeting therapeutic agents}

It has been reported in previous studies that porphyrins can be rapidly and preferentially taken up by cancer cells [30, 31]. Rapidly proliferating cells, such as cancer cells, provide opportunities for the porphyrins to interact with $G$ quadruplexes on single-stranded (ss) DNA [32]. This tumor selectivity of porphyrin and its derivatives, together with their photodynamic action, has eventually enabled these porphyrin compounds to be used in clinical applications as sensors for photodynamic therapy for various cancers [33, 34]. As gold (III) porphyrin 1a exhibits a higher cytotoxicity towards tumor (SUNE1) cells than towards normal cells (NP 69), it is reasonable to expect that this porphyrin containing agent may also exhibit tumor selectivity in vivo.

\subsection{Mitochondria-mediated apoptotic pathway}

Apoptosis is a process of gene-mediated programmed cell death essential for the elimination of unwanted cells in various biological systems. One of the two major apoptotic pathways is the mitochondria-mediated pathway. Mitochondria play a crucial role in apoptosis by releasing several apoptogenic molecules, including cytochrome $c$ and Smac/ Diablo. They are released from the intermembrane space of mitochondria into the cytoplasm in response to a variety of death-promoting stimuli [35-37]. The release of cytochrome $c$ from mitochondria is mediated by VDAC1. VDAC1 is a mitochondrial outer membrane channel protein, which functions as the pathway for the movement of various substances in and out of the mitochondria [38]. It is considered to be a component of the permeability transition pore oligoprotein complex that plays a role in the permeability transition [39, 40]. VDAC1 also plays an essential role in Bax/Bakinduced apoptotic mitochondrial changes in the process of apoptosis in mammalian cells [41-43]. In this process, the pro-apoptotic proteins Bax and Bak bind to VDAC1, and enhance its permeability so that cytochrome $c$ passes through the channel and releases to cytoplasm [41-43]. Our

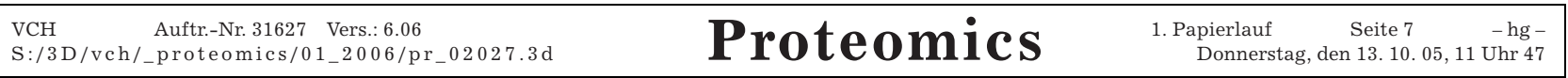




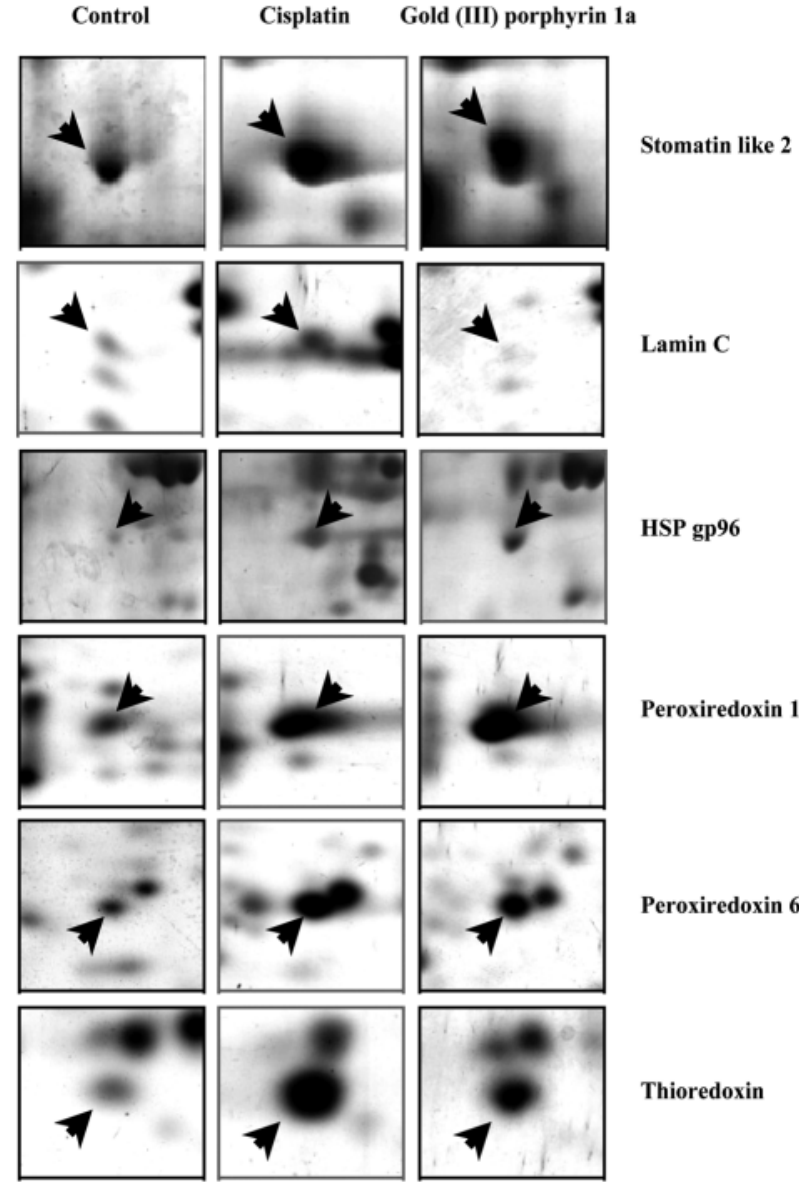

Figure 6. Detailed alteration patterns of cellular structure protein, chaperone proteins and proteins related to oxidative stress, including stomatin-like 2, lamin $C$, heat shock protein gp96 (HSPgp96), Prx 1 and 6, and thioredoxin.

data on VDAC1 up-regulation (Fig. 8) and Bax over-expression (to be published) suggest that gold (III) porphyrin 1a may induce cell death via the mitochondria-mediated apoptosis pathway.

Interestingly, we found that the related protein $\mathrm{mtSSB}$ was suppressed in SUNE1 cells treated with gold (III) porphyrin 1a (Fig. 7). mtSSB is a conserved molecule that binds to ssDNA, the template for the synthesis of lagging DNA strand synthesis mitochondrial DNA (mtDNA) replication, and prevention of mtDNA renaturation [44, 45]. The downregulation of $\mathrm{mtSSB}$ expression by gold (III) porphyrin 1a may destabilize mtDNA during replication, and further lead to apoptosis. These data again confirm that gold (III) porphyrin 1a may induce mitochondria-mediated apoptosis. Similarly, studies have demonstrated that cisplatin triggers apoptosis through the mitochondrial pathway in several cancer cell lines [46, 47]. Both gold (III) porphyrin 1a and cisplatin altered the expression of VDAC1 and mtSSB, suggesting that they may share the same mitochondrial apoptotic pathway.

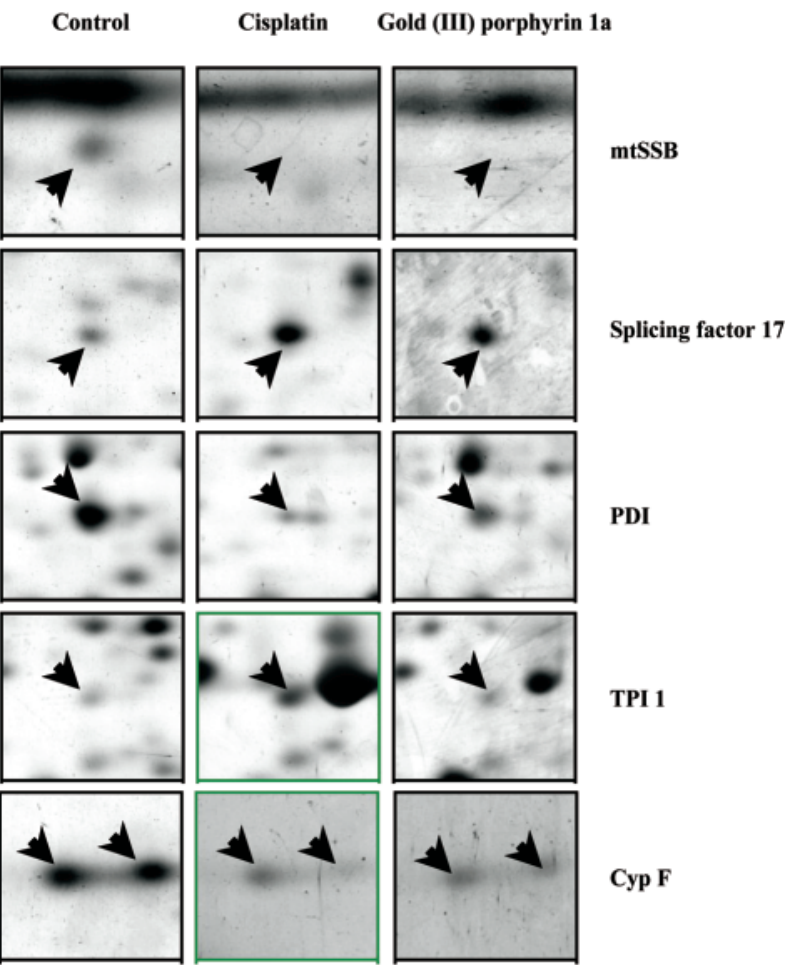

Figure 7. Detailed alterations of translation factors and enzyme proteins, including $\mathrm{mtSSB}$, splicing factor 17, PDI, triosephosphate isomerase 1 (TPI1), and cyclophilin F (CypF).

\subsection{Induction of ROS-related proteins}

Gold (III) porphyrin 1a induced alterations of the proteins involved in defending oxidative stress by up-regulating the expressions of thioredoxin, peroxiredoxin 1 , and peroxiredoxin 6 (Fig. 6). Alterations in cellular oxidative status have been shown to play an important role in inducing apoptotic cell death [36, 48, 49]. Low levels of ROS are required in the regulation of a broad range of normal cellular responses, including proliferation and cell survival [50-52]. Oxidative modifications of redox-sensitive transcription factors such as AP-1, transcription factor NF- $\kappa$ B , HIF- $1 \alpha$, and intermediate signaling molecules such as protein kinase C, ERK, and JNK, participate in this type of process $[50,53]$. On the other hand, high levels of ROS induce a state of oxidative stress in cells, which may damage cellular DNA, proteins, and lipids, and result in cell-cycle arrest, cellular senescence, and cell death [50]. The degree of oxidative stress and final cell fate are dependent on the balance between ROS anabolism and catabolism together with other factors. Cells are equipped with multiple antioxidant defense mechanisms for survival [54]. Among them, two important defense mechanisms were a thiol reducing buffer consisting of small proteins with redoxactive sulfhydryl moieties like glutathione (GSH) and thioredoxin; and enzymatic systems including superoxide dismutase, catalase, and glutathione peroxidase [55].

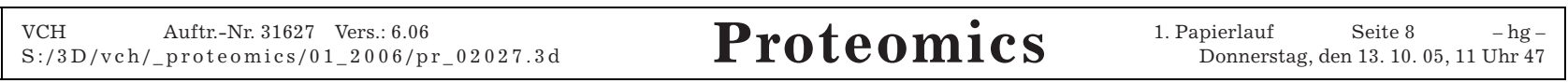




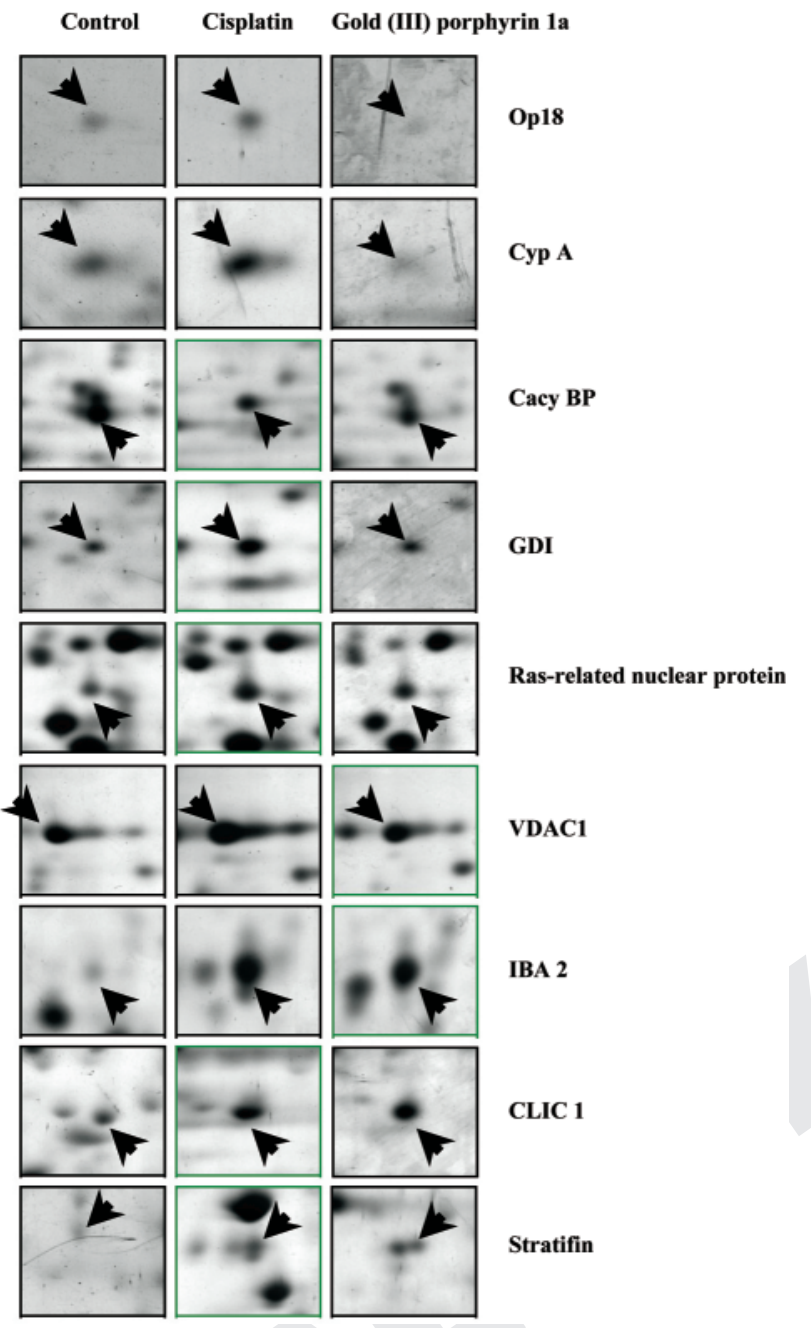

Figure 8. Detailed alteration patterns of proteins participating in cell proliferation or differentiation. This group of proteins contains Op18, СypA, CacyBP, GDI, Ras-related nuclear protein, VDAC1, IBA 2, CLIC 1, and stratinfin.

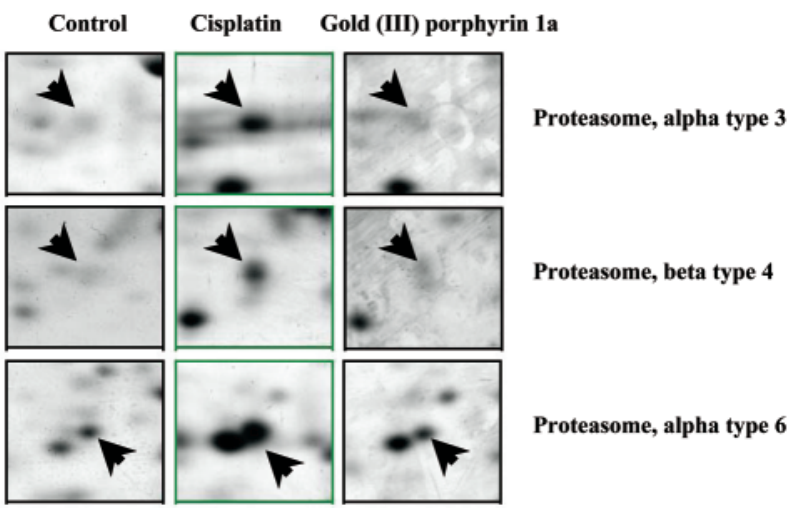

Figure 9. Detailed alteration patterns of proteins involved in the internal degradation system, including proteasome subunit alpha type 3 and type 6 , and beta type 4 .
Thioredoxin is a multifunctional and ubiquitous protein characterized by having a redox-active disulfide/dithiol within the conserved active site sequence: -Trp-Cys-Gly-ProCys-Lys- [56]. Previous studies have revealed that thioredoxin exerts a protective effect on cells exposed to cisplatin, and its over-expression is implicated in the mechanism of cisplatin resistance [57]. Our results (Fig. 7) also showed that thioredoxin was highly up-regulated in cells treated by cisplatinand in gold (III) porphyrin 1a-treated cells, which suggests that gold (III) porphyrin 1a may induce oxidative stress in cancer cells.

Peroxiredoxins (Prx) are a group of recently characterized thiol-containing proteins with efficient antioxidant capacity, capable of antagonizing hydrogen peroxide in living cells. It has been reported that Prx enzymes catalyze the reduction of hydrogen peroxide to water and alkyl hydroperoxides to the corresponding alcohols [58]. The Prx also influence a variety of cellular processes that are sensitive to ROS and play a role in signal transduction and gene expression related to alterations in cellular ROS levels [59]. Increased Prx expression correlated with chemoresistance by cisplatin treatment $[60$, 61]. Our results further suggest that gold (III) porphyrin 1a may induce ROS, which may subsequently alter mitochondrial membrane potential and ultimately induce mitochondria-mediated apoptosis. Similar findings have been reported in respect of cisplatin-induced apoptosis in various cancer cell lines [46, 47].

PDI was down-regulated in SUNE1 cells treated with both gold (III) porphyrin 1a and cisplatin (Fig. 7). PDI is a multifunctional protein mainly located in the endoplasmic reticulum (ER). During protein folding in the ER, PDI catalyzes thiol/disulfide exchange, including disulfide bond formation and rearrangement reactions [62]. The thiol/disulfide centers of the two thioredoxin-like domains function as two independent active sites. These two sites could lead to increased PDI expression in neurons, and result in both attenuation of the loss of cell viability in vitro and reduction of the number of DNA-fragmented cells in the rat hippocampal CA1 sub region in vivo [62]. Recently, Nomura and colleagues $[63,64]$ reported that up-regulated PDI may exert a protective effect against apoptotic cell death. The observed down-regulated expression of PDI in our study indicates that gold (III) porphyrin 1a triggers cancer cells towards apoptosis instead of proliferation.

\subsection{Altered proteins associated with cell proliferation or differentiation}

CacyBP, a novel protein target of calcyclin involved in calcium signaling pathways in neuronal tissue, was up-regulated in proliferating and differentiating cells [65]. Downregulation of CacyBP expression by gold (III) porphyrin 1a treatment (Fig. 8) suggests that gold (III) porphyrin 1a may induce cell apoptosis through affecting the processes of cell proliferation and differentiation.

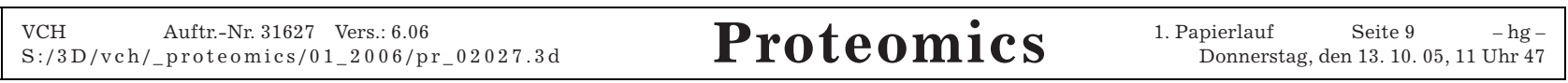



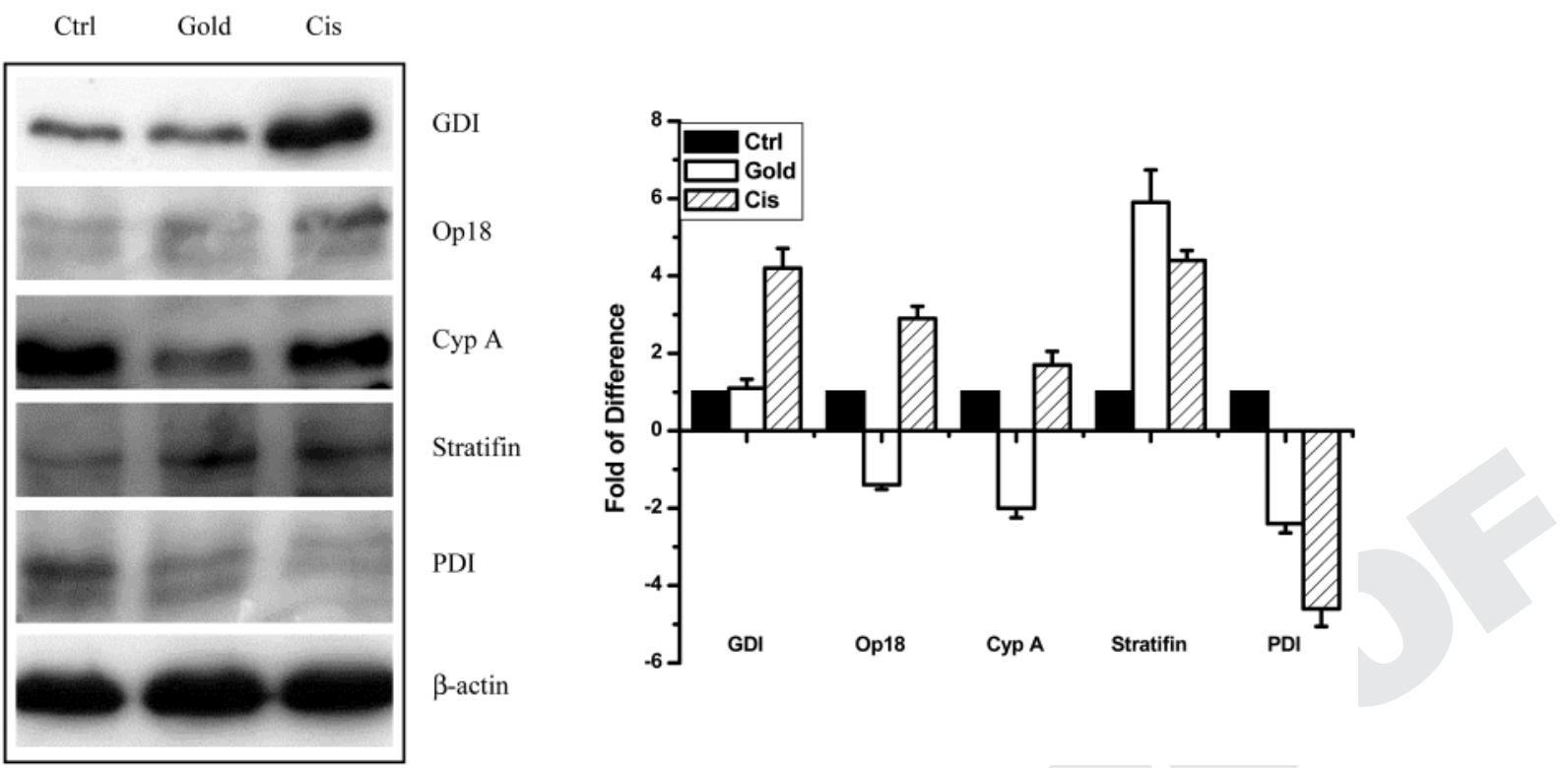

Figure 10. Western blot analysis of the altered proteins in gold (III) porphyrin 1a-treated SUNE1 cells. The altered expression ratio of GDI, Op18, Cyp A, stratifin and PDI was normalized to the intensity of $\beta$-actin, and presented as a chart.

CLIC 1 is a transmembrane protein that forms an essential component of an ion channel complex. Expression of CLIC 1 in cultured cells has been reported to lead to increased chloride channel activity in plasma and nuclear membranes $[66,67]$. Besides its novel role as a typical integral membrane protein, studies have also demonstrated that chloride ion (Cl-) conductance changes in synchrony with the cell cycle in a number of different cells [68]. Expression of a $\mathrm{Cl}^{-}$current was markedly up-regulated after cell cycle arrest, with the highest $\mathrm{Cl}^{-}$channel activity in early $\mathrm{G} 1$ and the lowest activity in G0/G1 and $\mathrm{S}$ phase $[69,70]$. The observed up-regulation of CLIC 1 in this study (Fig. 8) is consistent with our previous data showing that gold (III) porphyrin 1a abrogated SUNE1 cell cycle at G1 and inhibited DNA synthesis (to be published).

\subsection{Alteration of proteins involved in the internal protein degradation system}

The proteins associated with internal protein degradation system, including proteasome subunit alpha type 3 and 6 , and beta type 4, are up-regulated by cisplatin, and do not alter significantly by gold (III) porphyrin 1a (Fig. 9). Proteasome is a multisubunit enzyme complex that was initially thought to be a mere recycler of damaged or misfolded proteins. However, this enzyme complex has recently been found to play a central role in the regulation of proteins that control cell cycle progression and apoptosis [71]. Inhibition of proteasome can stabilize various cell cycle-regulatory proteins, including cyclin-dependent kinase inhibitors (CDKI) (e.g., p21 and p27), tumor suppressors (e.g., p53), and the transcription factor NF-кB [72-75]. Interference of proteasome function in a proliferating cell can therefore set cell arrest at the G0/G1 or G2 phase, leaving the cell with three alternatives: to remain in the G0/G1 or G2 phase, to follow a differentiation pathway, or to undergo apoptosis [76]. The upregulation of the proteasome has also been shown to be one of the key factors leading to drug resistance in chemotherapy [77].

\subsection{Concluding remarks}

Based on our findings in this study, we conclude that treatment with gold (III) porphyrin 1a caused multiple effects leading to apoptosis in SUNE1 cells. The balance between pro-apoptotic and anti-apoptotic signals determines the final fate of cancer cell survival. The general differences between the expressed proteomes implies that gold (III) porphyrin 1a may exert its cytotoxicity on cisplatin-resistant cancer cell lines. There is therefore a good chance that gold (III) porphyrin 1a could be successfully used in the treatment of cisplatin-resistant patients. Clearly, this study is only the beginning of a series of investigations, and further functional studies are needed to understand the overall action mechanisms of gold (III) porphyrin 1a.

The authors are indebted to Ms Yuan Zhou for her valuable technical assistance, and to Dr. David Wilmshurst for critically reading and editing this manuscript. This work was partially

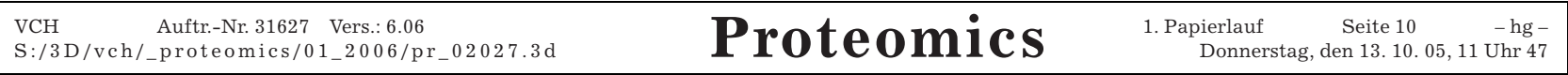


supported by grants from the Hong Kong Research Grant Council (grant no. 10204562 to J.F.C.), the University of Hong Kong's Seed Funding for Basic Research scheme (grant no. 10204407 to Q.Y.H.), and the Hong Kong University Grants Committee's Area of Excellence scheme.

\section{References}

[1] Kovarik, J., Svec, F., Thurzo, V., Neoplasma 1972, 19, 569577.

[2] Lippman, A. J., Helson, C., Helson, L., Krakoff, I. H., Cancer Chemother. Rep. 1973, 57, 191-200.

[3] Shaw, C. F., in: Fricker, S. P., (Ed.), Metal Compounds in Cancer Therapy, Chapman and Hall, London, 1994, pp. 46-64.

[4] Haiduc, I., Silvestru, C., In Vivo 1989, 3, 285-293.

[5] Sadler, P. J., Nasr, M., Narayanan, V. L., in: Hacker, M. P., Douple, E. B., Krakoff, I. H., (Ed.), Platinum Coordination Complexes in Cancer Chemotherapy, Martinus Nijhoff Publishing, Boston, 1984, pp. 209-304.

[6] Che, C. M., Sun, R. W., Yu, W. Y., Ko, C. B. et al., Chem. Commun. (Camb.) 2003, 14, 1718-1719.

[7] Kaufmann, S. H., Cancer Res. 1989, 49, 5870-5878.

[8] Barry, M. A., Behnke, C. A., Eastman, A., Biochem. Pharmacol. 1990, 40, 2353-2362.

[9] Youn, C. K., Kim, M. H., Cho, H. J., Kim, H. B. et al., Cancer Res. 2004, 64, 4849-4857.

[10] Schenk, P. W., Brok, M., Boersma, A. W., Brandsma, J. A. et al., Mol. Pharmacol. 2003, 64, 259-268.

[11] Kaufmann, S. H., Earnshaw, W. C., Exp. Cell Res. 2000, 256, 42-49.

[12] Hamilton, R. F. Jr, Li, L., Felder, T. B., Holian, A., Am. J. Physiol. 1995, 269, L318-L325.

[13] Muller, M., Strand, S., Hug, H., Heinemann, E. M. et al., J. Clin. Invest. 1997, 99, 403-413.

[14] Vaux, D. L., Korsmeyer, S. J., Cell 1999, 96, 245-254.

[15] Ferreira, C. G., Span, S. W., Peters, G. J., Kruyt, F. A. et al., Cancer Res. 2000, 60, 7133-7141.

[16] Rathmell, J. C., Thompson, C. B., Annu. Rev. Immunol. 1999, $17,781-828$.

[17] Budihardjo, I., Oliver, H., Lutter, M., Luo, X. et al., Annu. Rev. Cell Dev. Biol. 1999, 15, 269-290.

[18] Danesi, R., de Braud, F., Fogli, S., de Pas, T. M. et al., Pharmacol. Rev. 2003, 55, 57-103.

[19] Wasinger, V. C., Corthals, G. L., J. Chromatogr. B Analyt. Technol. Biomed. Life Sci. 2002, 771, 33-48.

[20] Liotta, L. A., Kohn, E. C., Petricoin, E. F., JAMA 2001, 286, 2211-2214.

[21] He, Q. Y., Chiu, J. F., J. Cell Biochem. 2003, 89, 868-886.

[22] Tang, W. P., Zeng, Y., Chinese J. Cancer 1983, 2, 70-72.

[23] Lau, A. T., He, Q. Y., Chiu, J. F., Biochem. J. 2004, 382, 641650.

[24] Mansouri, A., Ridgway, L. D., Korapati, A. L., Zhang, Q. et al., J. Biol. Chem. 2003, 278, 19245-19256.

[25] He, Q. Y., Lau, G. K., Zhou, Y., Yuen, S. T. et al., Proteomics $2003,3,666-674$
[26] Lee, S. I., Brown, M. K., Eastman, A., Biochem. Pharmacol. 1999, 58, 1713-1721.

[27] Qin, L. F., Ng, I. O., Cancer Lett. 2002, 175, 27-38.

[28] Fischer, S. J., McDonald, E. S., Gross, L., Windebank, A. J., Neurobiol. Dis. 2001, 8, 1027-1035.

[29] Orlandi, L., Colella, G., Bearzatto, A., Abolafio, G. et al., Eur. J. Cancer 2001, 37, 649-659.

[30] Georgiou, G. N., Ahmet, M. T., Houlton, A., Silver, J. et al., Photochem. Photobiol. 1994, 59, 419-422.

[31] Villanueva, A., Jori, G., Cancer Lett. 1993, 73, 59-64.

[32] Rha, S. Y., Izbicka, E., Lawrence, R., Davidson, K. et al., Clin. Cancer Res. 2000, 6, 987-993.

[33] Ali, H., van Lier, J. E., Chem. Rev. 1999, 99, 2379-2450.

[34] Dougherty, T. J., Gomer, C. J., Henderson, B. W., Jori, G. et al., J. Natl. Cancer Inst. 1998, 90, 889-905.

[35] Adams, J. M., Cory, S., Science 1998, 281, 1322-1326.

[36] Green, D. R., Reed, J. C., Science 1998, 281, 1309-1312.

[37] Tsujimoto, Y., Shimizu, S., FEBS Lett. 2000, 466, 6-10.

[38] Colombini, M., J. Membr. Biol. 1989, 111, 103-111.

[39] Bernardi, P., Broekemeier, K. M., Pfeiffer, D. R., J. Bioenerg. Biomembr. 1994, 26, 509-517.

[40] Zoratti, M., Szabo, I., Biochim. Biophys. Acta 1995, 1241, 139-176.

[41] Shimizu, S., Narita, M., Tsujimoto, Y., Nature 1999, 399, 483487.

[42] Madesh, M., Hajnoczky, G., J. Cell Biol. 2001, 155, 10031015.

[43] Shimizu, S., Shinohara, Y., Tsujimoto, Y., Oncogene 2000 , 19, 4309-4318.

[44] Van Tuyle, G. C., Pavco, P. A., J. Cell Biol. 1985, 100, 251-257.

[45] Eliseev, R. A., Gunter, K. K., Gunter, T. E., Exp. Cell Res. 2003, 289, 275-281.

[46] Henkels, K. M., Turchi, J. J., Cancer Res. 1999, 59, 3077-3083.

[47] Park, M. S., De Leon, M., Devarajan, P., J. Am. Soc. Nephrol. 2002, 13, 858-865.

[48] Armstrong, J. S., Steinauer, K. K., Hornung, B., Irish, J. M. et al., Cell Death Differ. 2002, 9, 252-263.

[49] Raha, S., Robinson, B. H., Am. J. Med. Genet. 2001, 106, 62 70.

[50] Martindale, J. L., Holbrook, N. J., J. Cell. Physiol. 2002, 192, 1-15.

[51] Burdon, R. H., Free Radic. Biol. Med. 1995, 18, 775-794.

[52] Davies, K. J., IUBMB Life 1999, 48, 41-47.

[53] Gopalakrishna, R., Jaken, S., Free Radic. Biol. Med. 2000, 28, 1349-1361.

[54] Mates, J. M., Perez-Gomez, C., Nunez de Castro, I., Clin. Biochem. 1999, 32, 595-603.

[55] Nakamura, H., Nakamura, K., Yodoi, J., Annu. Rev. Immunol. 1997, 15, 351-369.

[56] Holmgren, A., Bjornstedt, M., Methods Enzymol. 1995, 252, 199-208.

[57] Sasada, T., Iwata, S., Sato, N., Kitaoka, Y. et al., J. Clin. Invest. 1996, 97, 2268-2276.

[58] Chae, H. Z., Robison, K., Poole, L. B., Church, G. et al., Proc. Natl. Acad. Sci. USA 1994, 91, 7017-7021.

[59] Lim, M. J., Chae, H. Z., Rhee, S. G., Yu, D. Y. et al., Gene 1998, $216,197-205$. 
[60] Chung, Y. M., Yoo, Y. D., Park, J. K., Kim, Y. T. et al., Anticancer Res. 2001, 21, 1129-1133.

[61] Yo, Y. D., Chung, Y. M., Park, J. K., Ahn, C. M. et al., Exp. Mol. Med. 2002, 34, 273-277.

[62] Lyles, M. M., Gilbert, H. F., Biochemistry 1991, 30, 613-619.

[63] Tanaka, S., Uehara, T., Nomura, Y., J. Biol. Chem, 2000, 275, 10388-10393.

[64] Ko, H. S., Uehara, T., Nomura, Y., J. Biol. Chem. 2002, 277, 35386-35392.

[65] Nowotny, M., Bhattacharya, S., Filipek, A., Krezel, A. M. et al., J. Biol. Chem. 2000, 275, 31178-31182.

[66] Valenzuela, S. M., Martin, D. K., Por, S. B., Robbins, J. M. et al., J. Biol. Chem. 1997, 272, 12575-12582.

[67] Tonini, R., Ferroni, A., Valenzuela, S. M., Warton, K. et al., FASEB J. 2000, 14, 1171-1178.

[68] Bubien, J. K., Kirk, K. L., Rado, T. A., Frizzell, R. A., Science $1990,248,1416-1419$.
[69] Ullrich, N., Sontheimer, H., Am. J. Physiol. 1997, 273, C1290C1297.

[70] Villaz, M., Cinniger, J. C., Moody, W. J., J. Physiol. 1995, 488, 689-699.

[71] Kisselev, A. F., Goldberg, A. L., Chem. Biol. 2001, 8, 739-758.

[72] Shah, S. A., Potter, M. W., McDade, T. P., Ricciardi, R. et al., J. Cell. Biochem. 2001, 82, 110-122.

[73] An, W. G., Hwang, S. G., Trepel, J. B., Blagosklonny, M. V., Leukemia 2000, 14, 1276-1283.

[74] Jeremias, I., Kupatt, C., Baumann, B., Herr, I. et al., Blood 1998, 91, 4624-4631.

[75] Wu, Y., Luo, H., Kanaan, N., Wu, J., J. Cell Biochem. 2000, 76 596-604.

[76] Drexler, H. C., Apoptosis 1998, 3, 1-7.

[77] Tsuruo, T., Naito, M., Tomida, A., Fujita, N. et al., Cancer Sci. 2003, 94, 15-21. 